\title{
IMPLICIT SCHEME OF THE FINITE DIFFERENCE METHOD FOR THE SECOND-ORDER DUAL PHASE LAG EQUATION
}

\author{
Ewa MaJChrzaK \\ Silesian University of Technology, Gliwice, Poland \\ e-mail: ewa.majchrzak@polsl.pl \\ BOHDAN MOCHNACKI \\ University of Occupational Safety Management, Katowice, Poland \\ e-mail: bmochnacki@wszop.edu.pl
}

\begin{abstract}
The second-order dual phase lag equation (DPLE) as a mathematical model of the microscale heat transfer is considered. It is known that the starting point determining the final form of this equation is the generalized Fourier law in which two positive constants (the relaxation and thermalization times) appear. Depending on the order of the generalized Fourier law expansion into the Taylor series, different forms of the DPLE can be obtained. As an example of the problem described by the second-order DPLE equation, thermal processes proceeding in the domain of a thin metal film subjected to a laser pulse are considered. The numerical algorithm is based on an implicit scheme of the finite difference method. At the stage of numerical modeling, the first, second and mixed order of the dual phase lag equation are considered. In the final part of the paper, examples of different solutions are presented and conclusions are formulated.
\end{abstract}

Keywords: microscale heat transfer, dual phase lag model, implicit scheme of finite difference method

\section{Introduction}

The Fourier heat conduction model is based on the assumption of instantaneous propagation of the thermal wave in the domain considered. Intuitively, this approach seems to be incorrect, but it has worked for solving a number of macroscopic heat conduction problems. However, it turned out that for certain non-typical materials with a complex internal structure, the Fourier model is insufficient (Roetzel et al., 2003). Even more, deviations from the real course of the process can be seen in the case of microscale heat transfer.

It is obvious that accumulating enough energy to transfer to the nearest neighborhood would take time in the process of heat transfer (Zhang, 2007). So, the lag time of the heat flux in relation to the temperature gradient referred to as "a relaxation time" was introduced by Cattaneo (1948) and Vernotte (1958), and the appropriate energy equation (a hyperbolic PDE) became known as the Cattaneo-Vernotte equation. In the recent years, the heat conduction model in which two delay times appear has become more and more popular. This model is called the dual-phase lag one (Zhang, 2007; Tzou, 2015). The starting point for considerations is the generalized form of the Fourier law, e.g. (Faghri et al., 2010; Smith and Norris, 2003). Depending on the number of terms in the Taylor series expansion of this law, different forms of the dual phase lag equation (DPLE) can be obtained (see Section 2). The lag times appearing in DPLE are called the relaxation time and the thermalization time. Some simple tasks described by this equation (supplemented by appropriate boundary and initial conditions) can be solved analytically, e.g. (Ciesielski, 2017a; Tang and Araki, 1999; Askarizadeh et al., 2017; Mohammadi- 
-Fakhar and Momeni-Masuleh, 2010). However, most of the practical problems have been solved using numerical methods. Examples of such solutions in the field of the microscale heat transfer may be the papers (Majchrzak and Mochnacki, 2014; Ciesielski, 2017b; Dai and Nassar, 2000; Mochnacki and Paruch, 2013; Chen and Beraun, 2001) concerning the first-order DPLE.

The similar problems have been considered for non-homogeneous (multilayered) domains. In this place, the papers (Majchrzak et al., 2009; Qiu et al., 1994; Al-Nimr et al., 2004; Wang et al., 2006,2008 ) can be (as the examples) mentioned. The correct form of the boundary conditions between subdomains (here, the macroscopic boundary conditions are often used, which is a significant simplification) can be found in (Ho et al., 2003) while the detailed mathematical considerations were shown in (Majchrzak and Kałuża, 2017). In turn, in the paper (Majchrzak and Mochnacki, 2016), the problem of stability condition (explicit scheme of the FDM) was analyzed.

The numerical solutions concerning the second-order DPLE (based on the finite difference method) are the subject of works prepared by Castro et al. (2016) and Deng et al. (2017). The similar problems are discussed in the paper presented, but the wider class of equations and the other numerical algorithm are taken into account.

The applications of DPLE in the scope of bioheat transfer will not be discussed here.

\section{Dual-phase lag model}

The following well known thermal diffusion equation is considered

$$
c \frac{\partial T(X, t)}{\partial t}=-\nabla \cdot \mathbf{q}(X, t)+Q(X, t)
$$

where $c$ is a volumetric specific heat, $\mathbf{q}$ is a heat flux vector, $Q$ is a capacity of the internal volumetric heat source, $X, t$ denote the geometrical co-ordinates and time.

The relationship between the heat flux $\mathbf{q}$ and the temperature gradient $\nabla T$ is given in the form of the generalized Fourier law (Zhang, 2007; Smith and Norris, 2003), namely

$$
\mathbf{q}\left(X, t+\tau_{q}\right)=-\lambda \nabla T\left(X, t+\tau_{T}\right)
$$

where $\lambda$ is thermal conductivity, $\tau_{q}$ and $\tau_{T}$ are the relaxation time and thermalization time, respectively. The relaxation time $\tau_{q}$ is the mean time for electrons to change their energy states, while the thermalization time $\tau_{T}$ is the mean time required for electrons and lattice to reach equilibrium.

Using the Taylor series expansions, the following second-order approximation of formula (2.2) can be taken into account

$$
\mathbf{q}(X, t)+\tau_{q} \frac{\partial \mathbf{q}(X, t)}{\partial t}+\frac{\tau_{q}^{2}}{2} \frac{\partial^{2} \mathbf{q}(X, t)}{\partial t^{2}}=-\lambda\left[\nabla T(X, t)+\tau_{T} \frac{\partial \nabla T(X, t)}{\partial t}+\frac{\tau_{T}^{2}}{2} \frac{\partial^{2} \nabla T(X, t)}{\partial t^{2}}\right]
$$

which means

$$
-\mathbf{q}(X, t)=\tau_{q} \frac{\partial \mathbf{q}(X, t)}{\partial t}+\frac{\tau_{q}^{2}}{2} \frac{\partial^{2} \mathbf{q}(X, t)}{\partial t^{2}}+\lambda \nabla T(X, t)+\lambda \tau_{T} \frac{\partial \nabla T(X, t)}{\partial t}+\lambda \frac{\tau_{T}^{2}}{2} \frac{\partial^{2} \nabla T(X, t)}{\partial t^{2}}
$$

From equation (2.4) it results that

$$
\begin{gathered}
-\nabla \cdot \mathbf{q}(X, t)=\tau_{q} \frac{\partial[\nabla \cdot \mathbf{q}(X, t)]}{\partial t}+\frac{\tau_{q}^{2}}{2} \frac{\partial^{2}[\nabla \cdot \mathbf{q}(X, t)]}{\partial t^{2}}+\nabla[\lambda \nabla T(X, t)] \\
+\tau_{T} \frac{\partial\{\nabla[\lambda \nabla T(X, t)]\}}{\partial t}+\frac{\tau_{T}^{2}}{2} \frac{\partial^{2}\{\nabla[\lambda \nabla T(X, t)]\}}{\partial t^{2}}
\end{gathered}
$$


The last dependence is introduced in to equation (2.1), and then

$$
\begin{gathered}
c \frac{\partial T(X, t)}{\partial t}=\tau_{q} \frac{\partial[\nabla \cdot \mathbf{q}(X, t)]}{\partial t}+\frac{\tau_{q}^{2}}{2} \frac{\partial^{2}[\nabla \cdot \mathbf{q}(X, t)]}{\partial t^{2}}+\nabla[\lambda \nabla T(X, t)] \\
+\tau_{T} \frac{\partial\{\nabla[\lambda \nabla T(X, t)]\}}{\partial t}+\frac{\tau_{T}^{2}}{2} \frac{\partial^{2}\{\nabla[\lambda \nabla T(X, t)]\}}{\partial t^{2}}+Q(X, t)
\end{gathered}
$$

Equation (2.1) can also be written as

$$
\nabla \cdot \mathbf{q}(X, t)=-c \frac{\partial T(X, t)}{\partial t}+Q(X, t)
$$

Putting equation (2.7) into (2.6), one obtains

$$
\begin{gathered}
c \frac{\partial T(X, t)}{\partial t}=\tau_{q} \frac{\partial}{\partial t}\left[-c \frac{\partial T(X, t)}{\partial t}+Q(X, t)\right]+\frac{\tau_{q}^{2}}{2} \frac{\partial^{2}}{\partial t^{2}}\left[-c \frac{\partial T(X, t)}{\partial t}+Q(X, t)\right] \\
+\nabla[\lambda \nabla T(X, t)]+\tau_{T} \frac{\partial\{\nabla[\lambda \nabla T(X, t)]\}}{\partial t}+\frac{\tau_{T}^{2}}{2} \frac{\partial^{2}\{\nabla[\lambda \nabla T(X, t)]\}}{\partial t^{2}}+Q(X, t)
\end{gathered}
$$

Assuming the constant value of the volumetric specific heat $c$, one has

$$
\begin{gathered}
c\left[\frac{\partial T(X, t)}{\partial t}+\tau_{q} \frac{\partial^{2} T(X, t)}{\partial t^{2}}+\frac{\tau_{q}^{2}}{2} \frac{\partial^{3} T(X, t)}{\partial t^{3}}\right]=\nabla[\lambda \nabla T(X, t)]+\tau_{T} \frac{\partial\{\nabla[\lambda \nabla T(X, t)]\}}{\partial t} \\
+\frac{\tau_{T}^{2}}{2} \frac{\partial^{2}\{\nabla[\lambda \nabla T(X, t)]\}}{\partial t^{2}}+Q(X, t)+\tau_{q} \frac{\partial Q(X, t)}{\partial t}+\frac{\tau_{q}^{2}}{2} \frac{\partial^{2} Q(X, t)}{\partial t^{2}}
\end{gathered}
$$

Additionally, for $\lambda=$ const the last equation takes form

$$
\begin{gathered}
c\left[\frac{\partial T(X, t)}{\partial t}+\tau_{q} \frac{\partial^{2} T(X, t)}{\partial t^{2}}+\frac{\tau_{q}^{2}}{2} \frac{\partial^{3} T(X, t)}{\partial t^{3}}\right]=\lambda \nabla^{2} T(X, t)+\lambda \tau_{T} \frac{\partial\left[\nabla^{2} T(X, t)\right]}{\partial t} \\
+\lambda \frac{\tau_{T}^{2}}{2} \frac{\partial^{2}\left[\nabla^{2} T(X, t)\right]}{\partial t^{2}}+Q(X, t)+\tau_{q} \frac{\partial Q(X, t)}{\partial t}+\frac{\tau_{q}^{2}}{2} \frac{\partial^{2} Q(X, t)}{\partial t^{2}}
\end{gathered}
$$

As previously mentioned, dual phase lag equation (2.10) is often simplified by omitting appropriate components. For example, in several works (e.g. Tzou, 1995) the second order Taylor expression of heat flux and the first order Taylor expression of the temperature gradient are applied to take into account the phase lagging behavior. Ignoring the inner heat source (as in Tzou, 1995), the governing equation of temperature based on the DPL model is the following

$$
c\left[\frac{\partial T(X, t)}{\partial t}+\tau_{q} \frac{\partial^{2} T(X, t)}{\partial t^{2}}+\frac{\tau_{q}^{2}}{2} \frac{\partial^{3} T(X, t)}{\partial t^{3}}\right]=\lambda \nabla^{2} T(X, t)+\lambda \tau_{T} \frac{\partial\left[\nabla^{2} T(X, t)\right]}{\partial t}
$$

It is also possible to consider the energy equation in the form (assuming that $Q(X, t)=0$ )

$$
c\left[\frac{\partial T(X, t)}{\partial t}+\tau_{q} \frac{\partial^{2} T(X, t)}{\partial t^{2}}\right]=\lambda \nabla^{2} T(X, t)+\lambda \tau_{T} \frac{\partial\left[\nabla^{2} T(X, t)\right]}{\partial t}+\lambda \frac{\tau_{T}^{2}}{2} \frac{\partial^{2}\left[\nabla^{2} T(X, t)\right]}{\partial t^{2}}
$$

The most popular DPLE results from the assumption that the first-order approximation of formula (2.2) is used, and then (e.g. Tang and Araki, 1999; Al-Nimr et al., 2004; Majchrzak and Mochnacki, 2014)

$$
c\left[\frac{\partial T(X, t)}{\partial t}+\tau_{q} \frac{\partial^{2} T(X, t)}{\partial t^{2}}\right]=\lambda \nabla^{2} T(X, t)+\lambda \tau_{T} \frac{\partial\left[\nabla^{2} T(X, t)\right]}{\partial t}+Q(X, t)+\tau_{q} \frac{\partial Q(X, t)}{\partial t}
$$

One can see that for $\tau_{T}=0$, DPLE (2.13) takes form of the Cattaneo-Vernotte equation, while for $\tau_{q}=0$ and $\tau_{T}=0$ the well known macroscopic Fourier equation is obtained. 
Taking into account the numerical examples presented in the final part of the paper, a modified form of the Neumann boundary condition must still be formulated, namely

$$
\begin{aligned}
& q_{b}(X, t)+\tau_{q} \frac{\partial q_{b}(X, t)}{\partial t}+\frac{\tau_{q}^{2}}{2} \frac{\partial^{2} q_{b}(X, t)}{\partial t^{2}} \\
& \quad=-\lambda\left[\mathbf{n} \cdot \nabla T(X, t)+\tau_{T} \frac{\partial[\mathbf{n} \cdot \nabla T(X, t)]}{\partial t}+\frac{\tau_{T}^{2}}{2} \frac{\partial^{2}[\mathbf{n} \cdot \nabla T(X, t)]}{\partial t^{2}}\right]
\end{aligned}
$$

where $\mathbf{n} \cdot \nabla T(X, t)$ denotes normal derivative and $q_{b}(X, t)$ is the known boundary heat flux. In the case of simplified forms of the DPLE, the appropriate components in condition (2.14) should be neglected.

\section{Formulation of the problem}

Thermal processes proceeding in a thin metal film subjected to laser pulse are considered. A $1 \mathrm{D}$ problem is analyzed (heat transfer in the direction perpendicular to the layer is taken into account). The front surface $x=0$ is irradiated by a laser pulse and according to (Tang and Araki, 1999; Kaba and Dai, 2005), the conductional heat transfer in the domain considered can be modeled using the DPLE in which the volumetric heat source $Q(x, t)$ is introduced. At the same time, for $x=0$ and $x=L$, the non-flux conditions should be assumed. The laser irradiation is described by the following source term

$$
Q(x, t)=\sqrt{\frac{\beta}{\pi}} \frac{1-R}{t_{p} \delta} I_{0} \exp \left[-\frac{x}{\delta}-\beta \frac{\left(t-2 t_{p}\right)^{2}}{t_{p}^{2}}\right]
$$

where $I_{0}$ is the laser intensity, $t_{p}$ is the characteristic time of the laser pulse, $\delta$ is the optical penetration depth, $R$ is the reflectivity of the irradiated surface, and $\beta=4 \ln 2$.

In the most general case, the following DPLE is considered::

- for $0<x<L$

$$
\begin{gathered}
\frac{\partial T(x, t)}{\partial t}+\tau_{q} \frac{\partial^{2} T(x, t)}{\partial t^{2}}+w_{q} \frac{\tau_{q}^{2}}{2} \frac{\partial^{3} T(x, t)}{\partial t^{3}}=a \frac{\partial^{2} T(x, t)}{\partial x^{2}}+a \tau_{T} \frac{\partial^{3} T(x, t)}{\partial t \partial x^{2}} \\
+w_{T} a \frac{\tau_{T}^{2}}{2} \frac{\partial^{4} T(x, t)}{\partial t^{2} \partial x^{2}}+\frac{1}{c} Q(x, t)+\frac{\tau_{q}}{c} \frac{\partial Q(x, t)}{\partial t}+w_{q} \frac{\tau_{q}^{2}}{2 c} \frac{\partial^{2} Q(x, t)}{\partial t^{2}}
\end{gathered}
$$

where $a=\lambda / c$ is the diffusion coefficient, $w_{T}$ and $w_{q}$ are bivalent parameters. Here $w_{T}=1$ and $w_{q}=1$. For the "simplified" forms of DPLE, they are equal to $(0,1),(1,0)$ and $(0,0)$.

As previously mentioned, $q_{b}(0, t)=q_{b}(L, t)=0$ and the appropriate boundary conditions are of the form (Eq. (2.14)):

- for $x=0$

$$
\frac{\partial T(x, t)}{\partial x}+\tau_{T} \frac{\partial^{2} T(x, t)}{\partial t \partial x}+w_{T} \frac{\tau_{T}^{2}}{2} \frac{\partial^{3} T(x, t)}{\partial t^{2} \partial x}=0
$$

— for $x=L$

$$
\frac{\partial T(x, t)}{\partial x}+\tau_{T} \frac{\partial^{2} T(x, t)}{\partial t \partial x}+w_{T} \frac{\tau_{T}^{2}}{2} \frac{\partial^{3} T(x, t)}{\partial t^{2} \partial x}=0
$$

The initial condition is also given for $t=0$

$$
T(x, 0)=\left.T_{p} \quad \frac{\partial T(x, t)}{\partial t}\right|_{t=0}=\left.u(x) \quad \frac{\partial^{2} T(x, t)}{\partial t^{2}}\right|_{t=0}=v(x)
$$

where $T_{p}$ is the initial temperature, while $u(x)$ and $v(x)$ are known functions. 


\section{Numerical algorithm}

The algorithm presented below is based on the implicit scheme of the finite difference method (FDM).

Let $T_{i}^{f}=T\left(x_{i}, f \Delta t\right)$, where $\Delta t$ is the time step, $x_{i}=i h$ ( $h$ is the geometrical mesh step) and $f=0,1, \ldots, F$. Taking into account initial conditions (3.5), on the assumption that $u(x)=$ $v(x)=0$, one has $T_{i}^{0}=T_{i}^{1}=T_{i}^{2}=T_{p}$. For the transition $t^{f-1} \rightarrow t^{f}(f \geqslant 3)$, the approximate form of equation (3.2) resulting from the introduction of adequate differential quotients is as follows

$$
\begin{aligned}
& \frac{T_{i}^{f}-T_{i}^{f-1}}{\Delta t}+\tau_{q} \frac{T_{i}^{f}-2 T_{i}^{f-1}+T_{i}^{f-2}}{(\Delta t)^{2}}+w_{q} \frac{\tau_{q}^{2}}{2} \frac{T_{i}^{f}-3 T_{i}^{f-1}+3 T_{i}^{f-2}-T_{i}^{f-3}}{(\Delta t)^{3}} \\
& \quad=a \frac{T_{i-1}^{f}-2 T_{i}^{f}+T_{i+1}^{f}}{h^{2}}+\frac{a \tau_{T}}{\Delta t}\left(\frac{T_{i-1}^{f}-2 T_{i}^{f}+T_{i+1}^{f}}{h^{2}}-\frac{T_{i-1}^{f-1}-2 T_{i}^{f-1}+T_{i+1}^{f-1}}{h^{2}}\right) \\
& \quad+w_{T} \frac{a \tau_{T}^{2}}{2(\Delta t)^{2}}\left(\frac{T_{i-1}^{f}-2 T_{i}^{f}+T_{i+1}^{f}}{h^{2}}-2 \frac{T_{i-1}^{f-1}-2 T_{i}^{f-1}+T_{i+1}^{f-1}}{h^{2}}+\frac{T_{i-1}^{f-2}-2 T_{i}^{f-2}+T_{i+1}^{f-2}}{h^{2}}\right) \\
& \quad+\frac{1}{c} Q_{i}^{f}+\frac{\tau_{q}}{c}\left(\frac{\partial Q}{\partial t}\right)_{i}^{f}+w_{q} \frac{\tau_{q}^{2}}{2 c}\left(\frac{\partial^{2} Q}{\partial t^{2}}\right)_{i}^{f}
\end{aligned}
$$

After mathematical transformations, one has

$$
\begin{aligned}
- & \frac{a\left[2(\Delta t)^{2}+2 \tau_{T} \Delta t+w_{T} \tau_{T}^{2}\right]}{2 h^{2}(\Delta t)^{2}} T_{i-1}^{f}+\left[\frac{2(\Delta t)^{2}+2 \tau_{q} \Delta t+w_{q} \tau_{q}^{2}}{2(\Delta t)^{3}}\right. \\
& \left.+\frac{2 a\left[2(\Delta t)^{2}+2 \tau_{T} \Delta t+w_{T} \tau_{T}^{2}\right]}{2 h^{2}(\Delta t)^{2}}\right] T_{i}^{f}-\frac{a\left[2(\Delta t)^{2}+2 \tau_{T} \Delta t+w_{T} \tau_{T}^{2}\right]}{2 h^{2}(\Delta t)^{2}} T_{i+1}^{f} \\
& =\frac{2(\Delta t)^{2}+4 \tau_{q} \Delta t+3 w_{q} \tau_{q}^{2}}{2(\Delta t)^{3}} T_{i}^{f-1}-\frac{2 \tau_{q} \Delta t+3 w_{q} \tau_{q}^{2}}{2(\Delta t)^{3}} T_{i}^{f-2} \\
& +\frac{w_{q} \tau_{q}^{2}}{2(\Delta t)^{3}} T_{i}^{f-3}-\frac{a \tau_{T}\left(\Delta t+w_{T} \tau_{T}\right)}{h^{2}(\Delta t)^{2}}\left(T_{i-1}^{f-1}-2 T_{i}^{f-1}+T_{i+1}^{f-1}\right) \\
& +\frac{a w_{T} \tau_{T}^{2}}{2 h^{2}(\Delta t)^{2}}\left(T_{i-1}^{f-2}-2 T_{i}^{f-2}+T_{i+1}^{f-2}\right)+\frac{1}{c} Q_{i}^{f}+\frac{\tau_{q}}{c}\left(\frac{\partial Q}{\partial t}\right)_{i}^{f}+w_{q} \frac{\tau_{q}^{2}}{2 c}\left(\frac{\partial^{2} Q}{\partial t^{2}}\right)_{i}^{f}
\end{aligned}
$$

Denoting

$$
\begin{aligned}
A= & -\frac{a\left[2(\Delta t)^{2}+2 \tau_{T} \Delta t+w_{T} \tau_{T}^{2}\right]}{2 h^{2}(\Delta t)^{2}} \quad B=\frac{2(\Delta t)^{2}+2 \tau_{q} \Delta t+w_{q} \tau_{q}^{2}}{2(\Delta t)^{3}}-2 A \\
C_{i}^{f} & =\frac{2(\Delta t)^{2}+4 \tau_{q} \Delta t+3 w_{q} \tau_{q}^{2}}{2(\Delta t)^{3}} T_{i}^{f-1}-\frac{2 \tau_{q} \Delta t+3 w_{q} \tau_{q}^{2}}{2(\Delta t)^{3}} T_{i}^{f-2}+\frac{w_{q} \tau_{q}^{2}}{2(\Delta t)^{3}} T_{i}^{f-3} \\
& -\frac{a \tau_{T}\left(\Delta t+w_{T} \tau_{T}\right)}{h^{2}(\Delta t)^{2}}\left(T_{i-1}^{f-1}-2 T_{i}^{f-1}+T_{i+1}^{f-1}\right)+\frac{a w_{T} \tau_{T}^{2}}{2 h^{2}(\Delta t)^{2}}\left(T_{i-1}^{f-2}-2 T_{i}^{f-2}+T_{i+1}^{f-2}\right) \\
& +\frac{1}{c} Q_{i}^{f}+\frac{\tau_{q}}{c}\left(\frac{\partial Q}{\partial t}\right)_{i}^{f}+w_{q} \frac{\tau_{q}^{2}}{2 c}\left(\frac{\partial^{2} Q}{\partial t^{2}}\right)_{i}^{f}
\end{aligned}
$$

one obtains

$$
A T_{i-1}^{f}+B T_{i}^{f}+A T_{i+1}^{f}=C_{i}^{f}
$$

The FDM equation resulting from the boundary condition for $x=0$ is of the form

$$
\begin{aligned}
& \frac{T_{1}^{f}-T_{0}^{f}}{h}+\frac{\tau_{T}}{\Delta t}\left(\frac{T_{1}^{f}-T_{0}^{f}}{h}-\frac{T_{1}^{f-1}-T_{0}^{f-1}}{h}\right) \\
& \quad+\frac{w_{T} \tau_{T}^{2}}{2(\Delta t)^{2}}\left(\frac{T_{1}^{f}-T_{0}^{f}}{h}-2 \frac{T_{1}^{f-1}-T_{0}^{f-1}}{h}+\frac{T_{1}^{f-2}-T_{0}^{f-2}}{h}\right)=0
\end{aligned}
$$


or

$$
\begin{array}{r}
-\left[2(\Delta t)^{2}+2 \tau_{T} \Delta t+w_{T} \tau_{T}^{2}\right] T_{0}^{f}+\left[2(\Delta t)^{2}+2 \tau_{T} \Delta t+w_{T} \tau_{T}^{2}\right] T_{1}^{f} \\
=\left(2 \tau_{T} \Delta t+2 w_{T} \tau_{T}^{2}\right)\left(T_{1}^{f-1}-T_{0}^{f-1}\right)-w_{T} \tau_{T}^{2}\left(T_{1}^{f-2}-T_{0}^{f-2}\right)
\end{array}
$$

Let us denote

$$
D=2(\Delta t)^{2}+2 \tau_{T} \Delta t+w_{T} \tau_{T}^{2} \quad E=2 \tau_{T} \Delta t+2 w_{T} \tau_{T}^{2}
$$

then

$$
-D T_{0}^{f}+D T_{1}^{f}=E\left(T_{1}^{f-1}-T_{0}^{f-1}\right)-w_{T} \tau_{T}^{2}\left(T_{1}^{f-2}-T_{0}^{f-2}\right)
$$

In a similar way, for $x=L$, one has

$$
-D T_{n-1}^{f}+D T_{n}^{f}=E\left(T_{n}^{f-1}-T_{n-1}^{f-1}\right)-w_{T} \tau_{T}^{2}\left(T_{n}^{f-2}-T_{n-1}^{f-2}\right)
$$

So, the final form of the system of equations corresponding to the transition $t^{f-1} \rightarrow t^{f}(f \geqslant 3)$ is the following

$$
\begin{aligned}
& -D T_{0}^{f}+D T_{1}^{f}=E\left(T_{1}^{f-1}-T_{0}^{f-1}\right)-w_{T} \tau_{T}^{2}\left(T_{1}^{f-2}-T_{0}^{f-2}\right) \\
& A T_{i-1}^{f}+B T_{i}^{f}+A T_{i+1}^{f}=C_{i}^{f} \quad i=1,2, \ldots, n-1 \\
& -D T_{n-1}^{f}+D T_{n}^{f}=E\left(T_{n}^{f-1}-T_{n-1}^{f-1}\right)-w_{T} \tau_{T}^{2}\left(T_{n}^{f-2}-T_{n-1}^{f-2}\right)
\end{aligned}
$$

So, the transition from $t^{f-1}$ to $t^{f}(f \geqslant 3)$ requires solving of the system of equations with a three-band main matrix which is the fastest solved using the Thomas algorithm.

\section{Examples of computations}

Thin metal films $(L=100 \mathrm{~nm})$ made of chromium, nickel and gold have been considered. The surface $x=0$ of the domain is subjected to the laser pulse. The parameters determining the capacity of the internal heat source (Eq. (3.1)) are equal to $I_{0}=13.7 \mathrm{~J} / \mathrm{m}^{2}, t_{p}=0.1 \mathrm{ps}$, $\delta=15.3 \mathrm{~nm}, R=0.93$. The initial temperature of the domain equals $T_{p}=300 \mathrm{~K}$, while the initial values of functions are $u(x)=0, v(x)=0$. Differential mesh parameters are $n=1000$, $\Delta t=0.0001 \mathrm{ps}$.

At the stage of numerical computations, constant values of thermophysical parameters have been assumed (mainly due to lack of other data in the literature) - see Table 1.

Table 1. Thermophysical parameters (Tzou, 2015)

\begin{tabular}{|l|c|c|c|}
\hline & Chromium & Gold & Nickel \\
\hline \hline$c\left[\mathrm{MJ} /\left(\mathrm{m}^{3} \mathrm{~K}\right)\right]$ & 3.21484 & 2.4897 & 4 \\
\hline$\lambda[\mathrm{W} /(\mathrm{mK})]$ & 93 & 315 & 90.8 \\
\hline$\tau_{q}[\mathrm{ps}]$ & 0.136 & 8.5 & 0.82 \\
\hline$\tau_{T}[\mathrm{ps}]$ & 7.86 & 90 & 10 \\
\hline
\end{tabular}

Computations have been performed in versions corresponding to $w_{T}=0, w_{q}=0$ (first-order DPLE), $w_{T}=1, w_{q}=1$ (second-order DPLE), $w_{T}=0, w_{q}=1$ and $w_{T}=1, w_{q}=0(\operatorname{mixed}$ order DPLE). Additionally, for comparative purposes, numerical solutions of the classical Fourier problems have been also found. The results are presented in the form of heating/cooling curves at the irradiated surface. The set of solutions for the chromium layer is shown in Fig. 1. For the other materials (Figs. 2 and 3), the solutions corresponding to the Fourier model, $w_{T}=0$, $w_{q}=0$ and $w_{T}=1, w_{q}=1$ are distinguished. The discussion of the results obtained will be carried out in the next Section. 


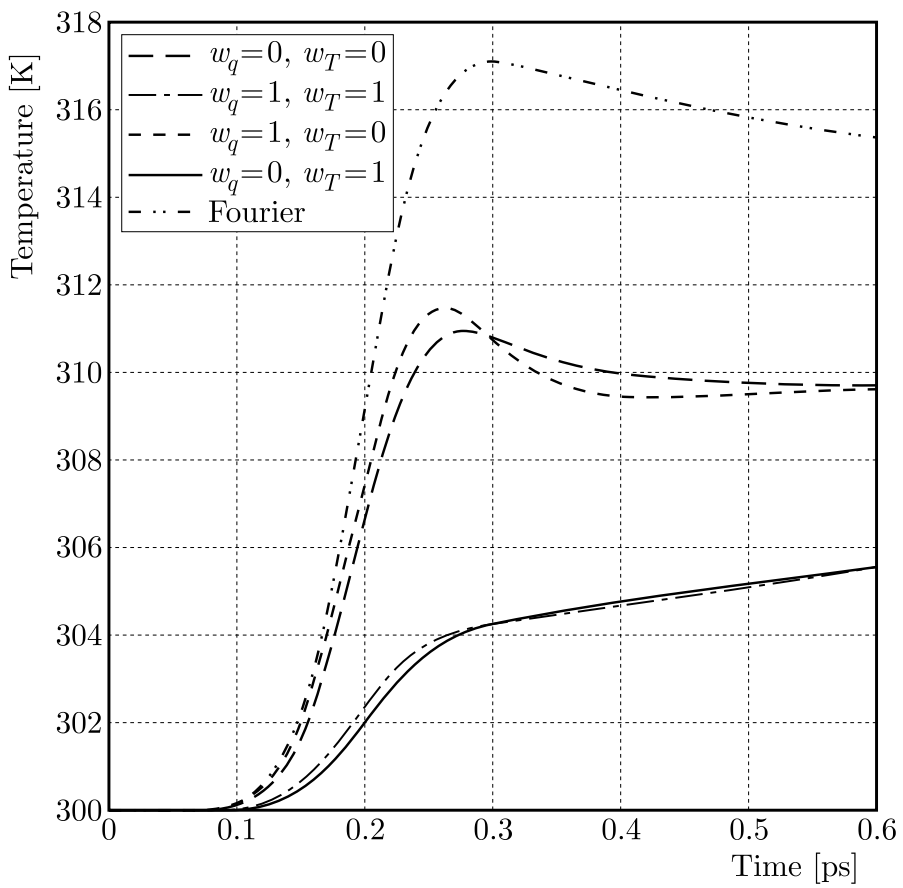

Fig. 1. Temperature history at the irradiated surface for different models (chromium)

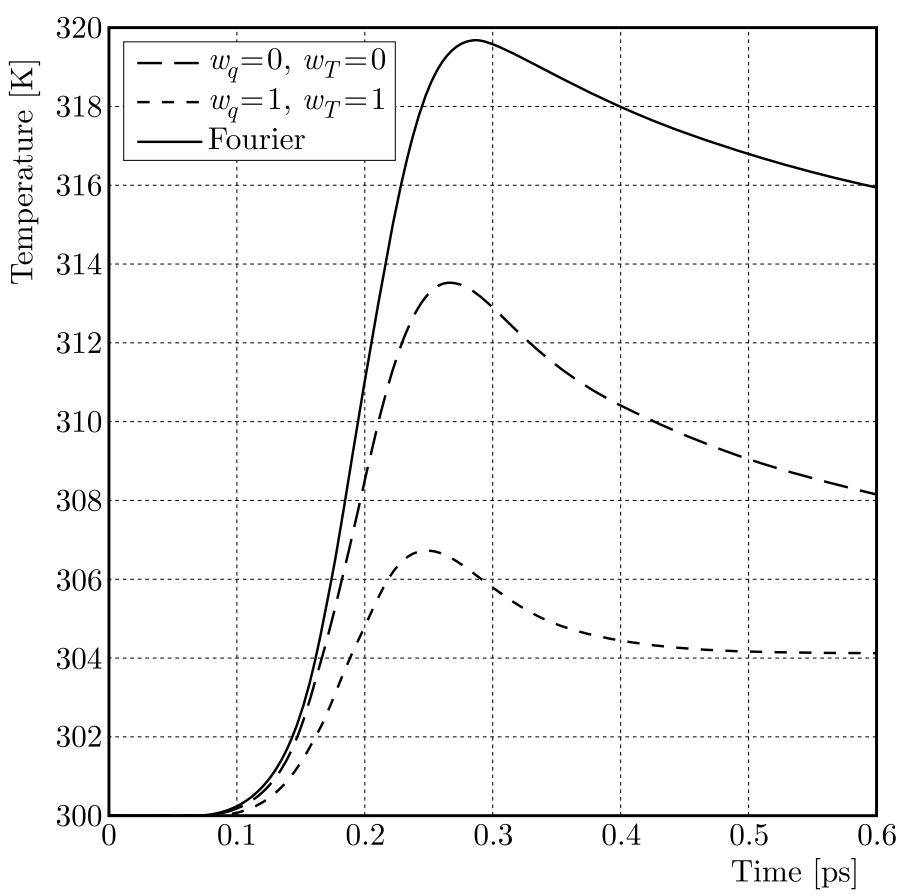

Fig. 2. Temperature history at the irradiated surface for different models (gold)

\section{Conclusions}

Different (in the sense of the order) models using the dual phase lag equation give different results. Here, one can see some regularities. In relation to the model based on the second-order DPLE, the solution resulting from the first-order equation is clearly overstated. This is the case for all the materials in question. The fact that the Fourier model gives a solution over DPLE has been repeatedly confirmed in numerous papers. This is a natural consequence of the delay 


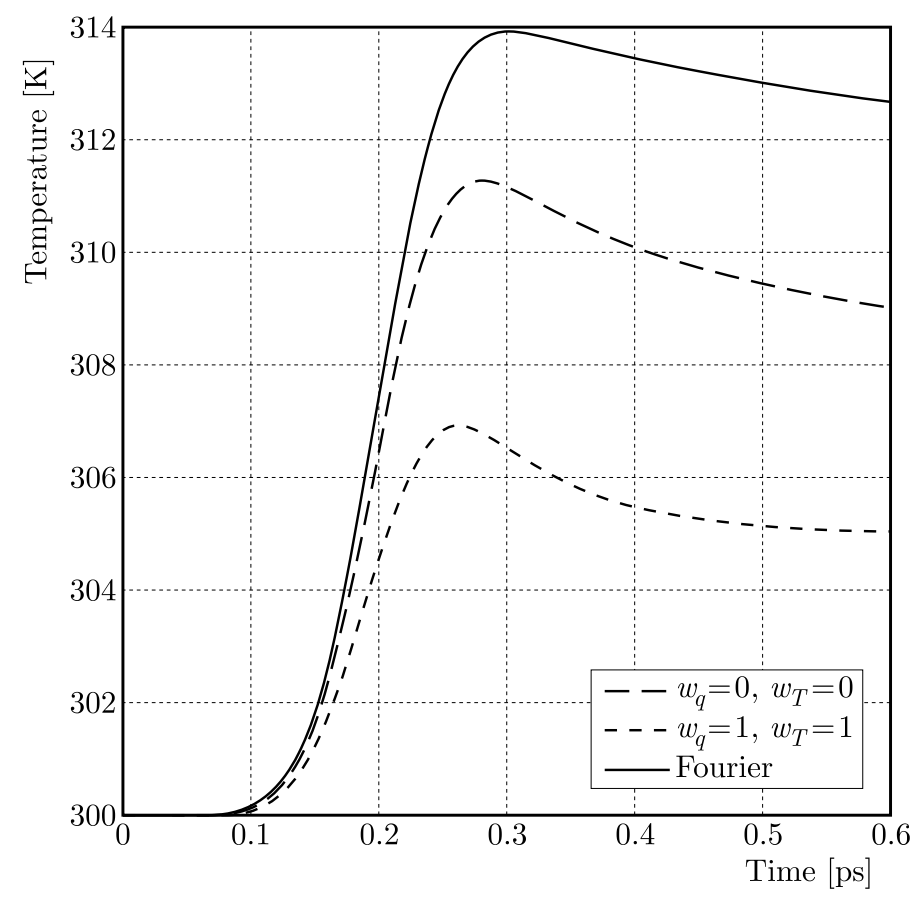

Fig. 3. Temperature history at the irradiated surface for different models (nickel)

times introduced. In the case of mixed models, the omission of the component containing $\tau_{T}^{2}$ (Eq. (2.11)) leads to results close to the solution of the first-order DPLE - see Fig. 1. On the other hand, the omission of the component containing $\tau_{q}^{2}$ (Eq. (2.12)) gives a solution similar to the solution of the second-order DPLE. The same trend is observed for the remaining materials. This results from the much larger (in the case of metals) value of the thermalization time versus the relaxation one. Therefore, more components of the Taylor series should be included on the right hand side of the generalized Fourier law. Summing up, the problems connected with the modeling of thermal processes in metal microdomains should be solved using the second-order dual phase lag equation. If the delay times vary less, then the solution based on the first-order model is sufficiently accurate.

\section{Acknowledgement}

The paper and research were financed within the project 2015/19/B/ST8/01101 sponsored by The National Science Centre (Poland).

\section{References}

1. Al-Nimr M.A., Naji M., AbDallah R.I., 2004, Thermal behavior of a multi-layered thin slab carrying periodic signals under the effect of the dual-phase-lag heat conduction model, International Journal of Thermophysics, 25, 3, 949-966

2. Askarizadeh H., Baniasadi E., Ahmadikia H., 2017, Equilibrium and non-eqilibrium thermodynamic analysis of high-order dual-phase lag heat conduction, International Journal of Heat and Mass Transfer, 104, 301-309

3. Castro M.A., Rodriguez F., Cabrera J., Martin, J.A., 2016, A compact difference scheme for numerical solution of second order dual-phase-lagging models of microscale heat transfer, Journal of Computational and Applied Mathematics, 201, 432-440

4. Cattaneo M.C., 1948, Sulla conduzione de calor, Atti de Seminario Matematico e Fisico Della Universita di Modena, 3, 3, 3-21 
5. Chen J. K., Beraun J. E., 2001, Numerical study of ultrashort laser pulse interactions with metal films, Numerical Heat Tranfer A - Applications, 40, 1-20

6. Ciesielski M., 2017a, Analytical solution of the dual phase lag equation describing the laser heating of thin metal film, Journal of Applied Mathematics and Computational Mechanics, 16, 1, $33-40$

7. Ciesielski M., 2017b, Application of the alternating direction implicit method for numerical solution of the dual phase lag equation, Journal of Theoretical and Applied Mechanics, 55, 3, $839-852$

8. Dai W., NAssar R., 2000, A compact finite difference scheme for solving a three-dimensional heat transport equation in a thin film, Numerical Methods for Partial Differential Equations, 16, 441-458

9. Deng D., JiAng Y., LiAng D. L., 2017, High-order finite difference methods for a second-order dual-phase-lagging models of microscale heat transfer, Applied Mathematics and Computation, 309, $31-48$

10. Faghri A., Zhang Y., Howell J., 2010, Advanced Heat and Mass Transfer, Global Digital Press

11. Ho A.J.R., Kuo Ch.P., Jiaung W.S., 2003, Study of heat transfer in multilayered structure within the framework of dual-phase-lag heat conduction model using lattice Boltzmann method, International Journal of Heat and Mass Transfer, 46, 55-69

12. KabA I., DAi W., 2005, A stable three-level finite difference scheme for solving the parabolic two-step model in a 3D micro-sphere heated by ultrashort-pulsed lasers, Journal of Computational and Applied Mathematics, 181, 125-147

13. Majchrzak E., KaŁuża G., 2017, Analysis of thermal processes occurring in the heated multilayered metal films using the dual-phase lag model, Archives of Mechanics, 69, 4-5, 275-287

14. Majchrzak E., Mochnacki B., 2014, Sensitivity analysis of transient temperature field in microdomains with respect to dual-phase-lag-model parameters, International Journal for Multiscale Computational Engineering, 12, 1, 65-77

15. Majchrzak E., Mochnacki B., 2016, Dual-phase lag equation. Stability conditions of a numerical algorithm based on the explicit scheme of the finite difference method, Journal of Applied Mathematics and Computational Mechanics, 15, 3, 89-96

16. Majchrzak E., Mochnacki B., Suchy J.S., 2009, Numerical simulation of thermal processes proceeding in a multi-layered film subjected to ultrafast laser heating, Journal of Theoretical and Applied Mechanics, 47, 2, 383-396

17. Mochnacki B., Paruch M., 2013, Estimation of relaxation and thermalization times in microscale heat transfer model, Journal of Theoretical and Applied Mechanics, 51, 4, 837-845

18. Mohammadi-Fakhar V., Momeni-Masuleh S. H., 2010, An approximate analytic solution of the heat conduction equation at nanoscale, Physics Letters A, 374, 595-604

19. Qiu T.Q., Juhasz T., Suarez C., Bron N.E., Tien C.L., 1994, Femtoscond laser heating of multi-layers metals. II Experiments, International Journal of Heat and Mass Transfer, 37, 17, $2799-2808$

20. Smith A. N., Norris P. M., 2003, Microscale Heat Transfer, Chapter 18, [In:] Heat Transfer Handbook, John Willey \& Sons

21. Roetzel W., Putra N., Das S.K., 2003, Experiment and analysis for non-Fourier conduction in materials with non-homogeneous inner structure, International Journal of Thermal Sciences, 42, $541-552$

22. TAng D.W., Araki N., 1999, Wavy, wavelike, diffusive thermal responses of finite rigid slabs to high-speed heating of laser-pulses, International Journal of Heat and Mass Transfer, 42, 855-860

23. Tzou D.Y., 1995, A unified field approach for heat conduction from macro- to micro-scales, Journal of Heat Transfer, ASME, 117, 1, 8-16 
24. Tzou D.Y., 2015, Macro to Microscale Heat Transfer: The Lagging Behavior, John Wiley \& Sons, Ltd.

25. Vernotte P., 1958, Les paradoxes de la theorie continue de l'equation de la chaleur, Comptes Rendus de l'Academie des Sciences, 246, 3154-3155

26. Wang H., Dai W., Melnik R., 2006, A finite difference method for studying thermal deformation in a double-layered thin film exposed to ultrashort pulsed lasers, International Journal of Thermal Sciences, 45, 1179-1196

27. Wang H., Dai W., Hewavitharana L.G., 2008, A finite difference method for studying thermal deformation in a double-layered thin film with imperfect interfacial contact exposed to ultrashort pulsed lasers, International Journal of Thermal Sciences, 47, 7-24

28. Zhang Z.M., 2007, Nano/Microscale Heat Transfer, McGraw-Hill 